2018-06-08

\title{
Oral Care of Palliative Care Patients - Carers' and Relatives' Experiences. A Qualitative Study.
}

\section{Bernardes Delgado, Maria do Carmo}

http://hdl.handle.net/10026.1/10540

10.1038/sj.bdj.2018.434

British Dental Journal

Nature Publishing Group

All content in PEARL is protected by copyright law. Author manuscripts are made available in accordance with publisher policies. Please cite only the published version using the details provided on the item record or document. In the absence of an open licence (e.g. Creative Commons), permissions for further reuse of content should be sought from the publisher or author. 


\section{INTRODUCTION}

Oral symptoms are amongst the most common problems palliative care patients face. ${ }^{1-4}$ Oral health complaints become increasingly prevalent with advancing illnesses. ${ }^{4}$ These range from active dental caries in $20 \%$ to $33 \%$ of palliative care individuals ${ }^{5}$ to candidiasis and dry mouth. ${ }^{4}$ Lee et al. ${ }^{6}$ and Wiseman ${ }^{7}$ state that the quality of life of terminally-ill patients is greatly affected by oral health problems and that these can precipitate further complications. Consequently it is argued that oral health needs, must be taken into account when planning for someone’s end-of-life care.,

The General Medical Council (GMC) in the UK provides a definition of end-of-life care: "People are approaching end of life when they are likely to die within the next 12 months. This includes people whose death is imminent (expected within a few hours or days) and those with advanced, progressive, incurable conditions; general frailty and co-existing conditions that mean they are expected to die within 12 months; existing conditions if they are at risk of dying from sudden acute crisis in their condition; life threatening acute conditions caused by sudden catastrophic events."10

Terminally-ill individuals are likely to be receiving palliative care to help managing and reducing their symptoms. ${ }^{11}$ Palliative care is "an approach that improves the quality of life of patients and their families facing the problem associated with life-threatening illness, through the prevention and relief of suffering by means of early identification and impeccable assessment and treatment of pain and other problems, physical, psychosocial and spiritual.",12

The World Health Organisation ${ }^{13}$ (WHO) issued guidelines in order to assist health workers, community care providers and family members to provide home-based palliative care. This includes preventative oral care such as tooth brushing and management of key symptoms 
such as painful mouth ulcers, pain on swallowing and dry mouth. However the advice given is limited and it is not clear whether it is evidence-based.

Similarly in England the National Institute for Health and Care Excellence ${ }^{4}$ (NICE) provides guidance regarding palliative oral care. Palliative oral care is "the management of patients with far-advanced disease where the oral cavity has been compromised, either directly by the disease or indirectly as a consequence of its treatment". ${ }^{2}$ Several sections of the document, such as self-care, dry-mouth management and oral pain, are pragmatic recommendations and based on expert opinion. The advice provided by $\mathrm{NICE}^{4}$ regarding oral care for terminally-ill patients is also based partly on expert opinion rather than evidence.

It is argued that the oral care delivered to palliative care patients is not always adequate. ${ }^{2}$ NHS Scotland ${ }^{9}$ have stated that oral care is often neglected for palliative care patients. Some argue this is due to inadequately trained staff, lack of resources and time pressure. ${ }^{2}$ Moreover the guidance available is frequently not based on evidence but on expert advice.

A review of the available literature showed that articles published in the palliative oral care field focused on the management and report of commonly occurring oral problems. ${ }^{7,14-17}$ Most authors presented similar conclusions. The oral cavity, in palliative care patients, is commonly the first site of discomfort and loss of function. ${ }^{1-3,5-8,14,15,18,19}$ Additionally it was argued that dental professionals should be playing a more active role in providing care for these individuals. ${ }^{5,8,14,17}$ Moreover some authors have stated that palliative oral care must be improved and standardised. ${ }^{1,3,6,15,18}$ Lastly, Kvalheim et al. ${ }^{2}$ reported that there is a certain degree of confusion surrounding palliative oral care. This fact can be explained by the lack of evidence on which to base procedures. ${ }^{2}$

$\mathrm{NICE}^{20}$ issued oral health guidelines for adults in care homes, where it recognised that research in oral health focuses primarily on clinical dental indices. The difference between 
dental professionals' research priorities and what the individuals receiving and providing oral care value is rarely taken into consideration. ${ }^{20}$ This suggests that the care provided may not be patient-centred.

Research on palliative oral care is scarce and the perspective of patients, relatives and carers has rarely been sought. Consequently the aim of this study is to explore the oral care experiences of palliative care patients, from the perspective of their relatives and/or carers. Blogs and discussion forums, on public internet sites, were used to access data. This approach was chosen to avoid causing additional burden to this already compromised population. Terminally-ill patients, who require support or cannot perform basic oral care procedures, are unlikely to be able to keep a blog themselves. Hence this study examined the relatives and/or carers' reports. 


\section{METHODS}

\section{Theoretical approach}

The aim of this research is to explore the experiences of relatives and/or carers of terminallyill patients regarding their oral care. The researcher adopted a contextualist epistemological position. The contextualist position, sits between essentialism and constructionism. ${ }^{21}$ It asserts that there is not a single reality, but that people make meaning of their experience and that social context also influences those meanings, while simultaneously retaining focus on the reality. ${ }^{21}$ A phenomenological approach was adopted to study the "lived" subjective experiences of the carers and/or relatives. The textual data, found in blogs and discussion forums, provided useful sources in exploring the hermeneutics of these peoples’ experiences. $^{22}$

\section{Ethical considerations}

The internet is undoubtedly the most comprehensive source of written material and easily accessed data. ${ }^{22}$ Data can be generated without researchers interacting directly with participants, which bypasses some of the ethical concerns. ${ }^{23}$ However despite blogs and discussion forums being in the public domain, some authors argue that consent is still required. ${ }^{23}$ Hence, for the purposes of this study, the researcher analysed data that was on public internet sites. All data collected and analysed were kept anonymous.

\section{Data collection}

The search strategy was developed with the help of an information specialist. An electronic search using “Google” and known blog platforms such as "Tumblr” and "Wordpress” was conducted on January $25^{\text {th }}$, 2017. The search terms used and search history can be found in Appendix1. 
Blogs and discussion forums were selected using the following inclusion criteria:

- Blogs and discussion forums available on public internet sites;

- Authors are relatives and/or carers of palliative care patients, not healthcare professionals;

- $\quad$ Authors are over 18 years old;

- $\quad$ Oral care must be mentioned, even if the author writes about other topics;

- Blogs and discussion forums are written in English.

The search strategy resulted in twenty blogs and discussion forums being retrieved. After the removal of duplicates nineteen blogs and discussion forums were considered potentially eligible. Two blogs and one discussion forum were excluded after full-text analysis. One of the excluded blogs was written by a healthcare professional and the other described the signs of imminent death:

“...his mouth open. Staring into space.”

A discussion forum for carers which did not mention oral care was excluded from the study.

Eight blogs and eight discussion forums met the inclusion criteria and were analysed (Figure1). All authors were relatives of palliative care patients. Eleven authors were also carers, one author was not a carer and it was not clear in four discussion forums. Seven of the eight blogs were written by females and seven were written in the USA. All palliative care patients mentioned in the blogs were terminally-ill and the deaths of seven were recounted within the blogs. Six of the eight discussion forums were started by females and all were written in the UK. Six of the eight palliative care patients mentioned were terminally-ill and the deaths of three were mentioned within the discussion forums. Demographic data are presented in Appendix2. 
Electronic search: tumblr.com, medium.com, wordpress.com, squarespace.com, squarepress.com,

blogger.com, wix.com, weebly.com and community.macmillan.org.uk

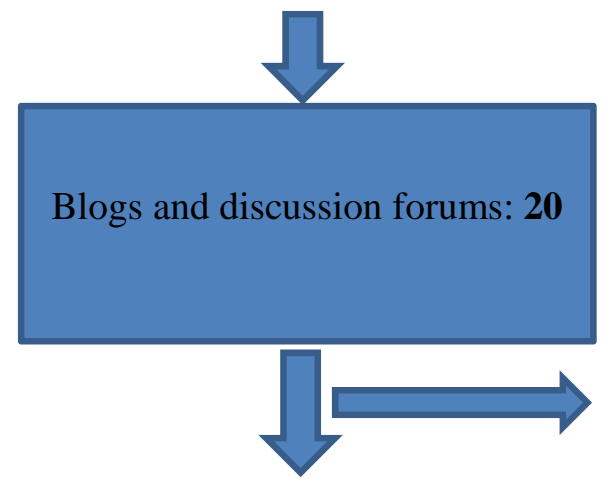

Duplicates removed: 1

Blogs and discussion forums: 19

10 blogs

9 discussion forums

Excluded: 3

2 blogs

- 1 written by healthcare professional

- 1 did not mention oral care

1 discussion forums

- 1 did not mention oral care

Blogs and discussion forums: 16

8 blogs

8 discussion forums

Figure1. Selection process of blogs and discussion forums 


\section{Data analysis}

The method chosen to analyse the data was thematic analysis. This method is not restricted to a single theoretical framework and can be used with a number of theoretical and epistemological positions, including a contextualist epistemological position and a phenomenological approach. ${ }^{23}$ It is also described as being a flexible foundational data analysis method, accessible to new researchers. ${ }^{23}$

Nonetheless Braun and Clarke ${ }^{23}$ offer guidance and the researcher followed the six phases described by these authors. XXX undertook a process of data immersion by reading each of the texts, in their entirety, several times and then focusing on the sections referring specifically to oral care. These sections were then extracted, retaining sufficient surrounding text to ensure comprehensiveness of meaning and initial codes were produced. Subsequently XXX sorted the different codes into potential themes. This resulted in candidate themes, multiple sub-themes and all the extracts of data that had been coded. The candidate themes were then refined. During this phase XXX reviewed all the extracts of data for each candidate theme. In addition XXX re-read the entire data set and a second reviewer (XXX), reviewed the themes, sub-themes and data extracts. This ensured rigour.

\section{FINDINGS}

Oral care was not the main topic in any of the blogs included and it was only the main topic in one of the discussion forums. Participant 12 described a specific oral symptom, dry mouth, and how to best address it. All authors mentioned oral care and the thematic analysis process, as described by Braun and Clarke ${ }^{23}$, identified three main themes about this topic:

\section{- Symptoms;}


- Procedures;

- Emotions.

\section{Symptoms}

Oral symptoms, such as dry mouth and difficulty in swallowing, were mentioned explicitly or implicitly in 15 of the 16 blogs and discussion forums. Dry mouth was explicitly mentioned by participant 1: “...swabbing my father's mouth with a little sponge dipped in solution would help with dryness...” and implicitly mentioned by participant 3: “They had provided sponges that we could use to moisten her lips and tongue...”.

The oral symptoms reported by most authors were dry mouth, dry lips and difficulty in swallowing. In one of the discussion forums (11), which reported on the side-effects of chemotherapy and radiotherapy, other symptoms were described such as loss of taste sensation, mouth ulcers and mucositis. These were symptoms relatives could observe themselves.

"My brother noticed a few hours before he died that his mouth was dry and he held a cotton wool swab dipped in water to his mouth...” (10)

“...his mouth is totally dry and obviously very unpleasant...” (12)

Therefore relatives could prepare for these symptoms. However, most authors were reacting to them.

"I would have watched for certain symptoms instead of reacting to them..." (8)

The oral symptoms palliative care patients experienced and that could be observed by their relatives, were often considered distressing to both patients and relatives. This was often an 
assumption on the part of the relatives. Fourteen of the sixteen palliative care patients mentioned in the blogs and discussion forums were terminally-ill.

"All I could do was put some lip balm on his lips and pray that it would offer him some relief to the dryness of his lips and he would forget about his dry mouth.” (5)

“...we were anxious to keep him as comfortable as possible so continued to offer the swab dipped in water.” (10)

Only one author (6) did not mention oral symptoms. The oral care provided to her father was part of his "morning cleaning”. This was his routine daily care and no oral symptoms were reported:

“This entailed giving him a sponge bath and changing his diaper, emptying his colostomy bag, cleaning out his mouth with a sponge and water..."

\section{Procedures}

All authors reported oral care procedures. Except for one of the blogs (1), these were described mainly as techniques. Eleven authors described the use of a swab/ sponge to clean and moisten the mouth:

“...swabbing my father's mouth with a little sponge dipped in solution...” (1).

In two blogs and one discussion forum, the authors reported using ice chips and/or frozen juice to moisten the mouth:

“...want to moisten their mouth, you can feed them tiny ice chips.” (2).

One blogger reported using lip balm to relief mouth dryness for her father. In one discussion forum, a named brand of chewy sweets are recommended to alleviate dry mouth. Most 
descriptions and recommendations did not provide much detail about the oral care procedure itself.

One discussion forum (11) provided more detailed advice on oral care. This was a discussion forum started by the wife of a cancer patient, inquiring on how to best help her husband through chemotherapy and radiotherapy. The participants of this forum gave very specific advice on what to do regarding loss of taste sensation, painful mouth ulcers, difficulty in swallowing and mucositis.

“... I used Difflam anaesthetic mouthwash to numb the ulcers for long enough to eat. It has to be taken 20 minutes before a meal...” (11)

In one of the blogs (1) the oral care procedures provided were more than a technical act to address oral symptoms. These were described as being simple procedures that were part of the regulations of the hospice. However the oral care was delivered at irregular times and was even considered dangerous:

"The little sponge should be squeezed and most of the solution or water drained out of it, then the person who is unable to swallow (my father)... will not aspirate liquid which could kill him. I turned my back... I saw another one pull the sponge from my father's mouth, dip it in the solution and reinsert it. My father began choking...” (1).

\section{Emotions}

When oral care procedures were described mainly as a technical act to address oral symptoms and as part of routine care, participants presented a neutral attitude and feelings towards them.

However some authors explored their emotions in relation to oral care more extensively. Participant 1 reported a general sense of anger towards the staff at the hospice. She described the oral care provided as being irregular, incorrect and even dangerous. 
“...sponge in a blue liquid, and heading towards my father's open mouth...I told her to get away from my father and the nurse supervisor came in and quickly removed the evidence..."

Participant 3 highlighted the revulsion and disgust felt by some of her family members when she was swabbing her grandmother's mouth.

"As I removed the sponge every other person in the room reacted with revulsion.” (3)

In addition she swabbed her grandmother's mouth to appease her family, who felt they had to do something to make her more comfortable.

Participants 10 and 13 expressed worry, guilt and trauma. Participant 10 was worried that her actions had caused her father unnecessary distress and there was guilt about this fact.

“...we were anxious to keep him as comfortable as possible so continued to offer the swab dipped in water. The problem is that I can't stop worrying about the fact that we might have caused him to choke.” (10)

Participant 13 described going through the trauma of witnessing their mother dying a painful death and feeling guilt about not having been able to do anything to help.

"Like you, I found it so hard to be unable to do anything for her at all, because I was told not to even swab her mouth to moisten it...” (13)

The emotions regarding oral care cannot be separated from the context in which these reports take place. Most palliative care patients mentioned are terminally-ill and the deaths of ten were recounted within the blogs and discussion forums. 


\section{DISCUSSION}

The objective of palliative care, including palliative oral care, is to provide relief from pain, stress and other symptoms. ${ }^{2}$ The aim is to improve quality of life for terminally-ill patients and their families. ${ }^{7}$

It is reported that the majority of palliative care patients present a wide range of oral symptoms. ${ }^{2,5,7,24}$ Data from Norway suggests that $56 \%$ of patients receiving end-of-life care complained of dry mouth, $49 \%$ complained of thirst and $74 \%$ of changes in eating habits. ${ }^{2}$ Dehydration is a common problem amongst these individuals.

Therefore it is not surprising that most participants described oral symptoms such as: dry mouth, dry lips and difficulty in swallowing. Oral diseases which can be symptomless and less easy to identify were not reported. However these findings are comparable to those by Rohr et al. ${ }^{15}$ In their study, Rohr et al. ${ }^{15}$ interviewed 14 terminally-ill patients about their experiences of oral discomfort and concluded dry mouth was the most commonly reported oral problem.

All participants described an oral care procedure, albeit without much detail. These were mainly a technical act to address oral symptoms and provide comfort. When oral care was not provided this was in order to avoid causing stress to their loved one and was usually because they had been advised not to do so. It appears that generally oral care was provided in reaction to signs and symptoms rather than to pro-actively improve quality of life.

The last theme that emerged from the data analysis, emotions, is not one that is prioritised in the papers or documents found during the literature reviewed in preparation for this study. The emotions described by the participants varied from anger, guilt, worry and trauma to a more neutral approach. When oral care was described as a technical act, participants did not 
report any positive or negative emotions. Some form of oral care was expected to happen and therefore this did not initiate an emotional response from the participants.

However when there was an emotional response to oral care, this was a negative one. One participant described the incompetence of staff providing oral care and this led to anger and stress. The fact that staff did not seem to know what to do was thought to have caused discomfort, pain and harm to the patient. This inevitably led to the relative feeling the emotions described above.

Furthermore when one of the participants swabbed her grandmother's mouth, other family members turned away or walked away expressing disgust, proving oral care is not seen as being easy or pleasant to perform for others. Indeed Couch et al. ${ }^{18}$ report similar findings in their study. They studied the oral care perceptions of paediatric palliative care nurses and described some of the barriers to providing mouth care, namely patient and/or family resistance.

Finally some participants felt guilt specifically in relation to oral care. There was guilt because they perceived that the oral care they provided was not correct and was potentially harmful. There was guilt for not being able to provide oral care or for being told not to do so.

\section{LIMITATIONS}

Blogs involve an enormous commitment in terms of time and energy. Therefore individuals that are terminally-ill and receive palliative care are very unlikely to be keeping a blog of their journey particularly in the latter stages of their illness. Hence we relied on reports from relatives for this study, which is a limitation. The relatives’ perspective and experience on oral care cannot be claimed to be that of the patients themselves. Relatives cannot speak for 
the patients and we also recognise that data was gathered in a context. It was not possible to assess the impact of context (e.g. UK vs USA) and other factors such as time of reporting and emotional state of the participants.

In addition as participants could not be probed, data collected may have less depth than for example data from interviews. Moreover the potential to get people to explore subjects that are more sensitive for them may have been missed as well. The analysis of the three themes, identified by using Braun and Clarke’s thematic analysis, could not be checked with the participants either. However a second experienced reviewer on qualitative research reviewed the themes, sub-themes and data extracts. This ensured rigour.

Finally the experiences of some participants were over-represented. This occurred as some participants wrote more about the topic of interest than others. Participants wrote about what was important to them rather than only about oral care. However the themes were supported by the analysis across all participants.

\section{CONCLUSION}

Terminally-ill individuals often present a multitude of oral symptoms. This inevitably compromises their quality of life. Therefore it is argued by healthcare professionals that oral care should be included in the ongoing care plan of every palliative care patient. However research on palliative oral care is scarce and the level of evidence underpinning it is weak. In addition the perspective of patients and relatives has rarely been sought.

This study aimed to begin to address this gap. Oral care was not the main topic in any of the blogs and most of the discussion forums and data on oral care were scarce. It seems, however, 
that oral care for terminally-ill patients tends to be overlooked until the person's quality of life becomes noticeably compromised by the oral symptomatology.

It cannot be claimed that the findings of this study are generalisable. Nonetheless they reflect the perspective of several individuals regarding the oral care provided to their terminally-ill relatives. This study did provide insight into a poorly researched area and will inform a future qualitative interview study.

\section{ACKNOWLEDGEMENTS}

XXX is an Academic Clinical Fellow, funded by the National Institute for Health Research (NIHR). 


\section{REFERENCES}

1. Kinley J, Brennan S. Changing practice: use of audit to change oral care practice. International Journal of Palliative Nursing. 2004;10:580-7.

2. Kvalheim SF, Strand GV, Husebo BS, Marthinussen MC. End-of-life palliative oral care in Norwegian health institutions. An exploratory study. Gerodontology. 2015:1-8.

3. Milligan S, McGill M, Sweeney MP, Malarkey C. Oral care for people with advanced cancer: an evidence-based protocol. International Journal of Palliative Nursing. 2001;7:41826.

4. NICE. Palliative care - oral 2016 [Available from: https://cks.nice.org.uk/palliative-careoral.

5. Saini R, PP M, Shete S, Saini S, Mani A. Dental expression and role in palliative treatment. Indian Journal of Palliative Care. 2009;15:26-9.

6. Lee L, White V, Gill K, Smart L, McEwan K, Chilton P, et al. An audit of oral care practice and staff knowledge in hospital palliative care. International Journal of Palliative Nursing. 2001;7:395-400.

7. Wiseman MA. Palliative care dentistry. Gerodontology. 2000;17:49-51.

8. Chen X, Chen H, Douglas C, Preisser JS, Shuman SK. Dental treatment intensity in frail older adults in the last year of life. JADA. 2013;144:1234-42.

9. NHS Scotland . Scottish Palliative Care Guidelines - Mouth Care 2014 [Available from: http://www.palliativecareguidelines.scot.nhs.uk/guidelines/symptom-control/mouth-care.aspx. 
10. GSF. The Gold Standards Framework Proactive Identification Guidance (PIG) 2016

[6th:[Available from: http://www.goldstandardsframework.org.uk/PIG.

11. Marie Curie. Marie Curie - Care and support through terminal illness 2016 [Available from: https://www.mariecurie.org.uk/help/support/diagnosed/recent-diagnosis/palliative-careend-of-life-care.

12. WHO. The sold facts: Palliative Care 2004 [Available from: http://www.euro.who.int/_data/assets/pdf_file/0003/98418/E82931.pdf?ua=1.

13. WHO. Palliative Care: symptom management and end-of-life care 2004 [Available from: http://www.who.int/hiv/pub/imai/genericpalliativecare082004.pdf.

14. Quinn B. Oral care for cancer and palliative patients. British Journal of Nursing. 2013;22:S14.

15. Rohr Y, Adams J, Young L. Oral discomfort in palliative care: results of an exploratory study of the experiences of terminally ill patients. International Journal of Palliative Nursing. 2010;16:439-44.

16. Sargeant S, Chamley C. Oral health assessment and mouth care for children and young people receiving palliative care. Part two. Nursing Children and Young People. 2013;25:30-3. 17. Wiseman MA. The treatment of oral problems in the palliative patient. J Can Dent Assoc. 2006;72:453-8.

18. Couch E, Mead JM, Walsh M. Oral health perceptions of paediatric palliative care nursing staff. International Journal of Palliative Nursing. 2013;19:9-15.

19. Croyere N, Belloir M, Chantler L, McEwan L. Oral care in nursing practice: a pragmatic representation. International Journal of Palliative Nursing. 2012;18:435-40. 
20. NICE. Oral health for adults in care homes 2016 [Available from:

https://www.nice.org.uk/guidance/ng48/resources/oral-health-for-adults-in-care-homes1837459547845.

21. Braun V, Clarke V. Using thematic analysis in Psychology. Qualitative Research in Phychology. 2006;3:77-101.

22. Keim-Malpass J, Steeves R, Kennedy C. Internet ethnography: A review of methodological considerations for studying online illness blogs. International Journal of Nursing Studies. 2014;51:1686-92.

23. Braun V, Clarke V. Successful qualitative research, a practical guide for beginners. London: Sage; 2013.

24. Jobbins J, Bagg J, Finlay IG, Addy M, Newcombe RG. Oral and dental disease in terminally ill cancer patients. BMJ. 1992;304:1612. 